\title{
Dendrocronología de Alnus acuminata en el bosque reservado de San Pedro de Saño, Huancayo
}

\author{
Alnus acuminata dendrochronology in the San Pedro de Saño \\ private forest, Huancayo
}

\author{
Edilson Jimmy Requena Rojas \\ Universidad Continental \\ edilson.requena@gmail.com
}

\section{RESUMEN}

En este trabajo evaluamos el potencial de Alnus acuminata para su uso posterior en estudios dendroclimatológicos. Se analizó una cronología de ancho de anillos para un bosque de A. acuminata en San Pedro de Saño y se relacionó el crecimiento de los árboles con los registros instrumentales de temperatura media, máxima y mínima, precipitación y horas sol de las estaciones meteorológicas de Huayao (12 $02^{\prime} 18.1^{\prime \prime} \mathrm{S}-75^{\circ} 19^{\prime} 22.2^{\prime \prime}$ O) y Santa Ana (12 $02^{\prime} 15^{\prime \prime} \mathrm{S}-75^{\circ} 13^{\prime} 15^{\prime \prime}$ O). La cronología de ancho de anillos está compuesta por 33 series de 22 árboles y cubre el período 1968-2013. El crecimiento de los árboles estuvo relacionado negativamente con la temperatura mínima mensual del mes de abril del año corriente. Se observó una relación positiva y significativa entre la cronología de crecimiento y la precipitación de la estación de Santa Ana para los meses de marzo del año previo y diciembre del año actual de crecimiento. Las horas sol mensuales de abril del año corriente tienen una correlación significativa con el crecimiento radial. Existe influencia de las precipitaciones del verano previo y primavera del año corriente favoreciendo el crecimiento del árbol. El árbol entra en dormancia, posiblemente por las bajas temperaturas de otoño. El amplio rango de distribución y la utilización histórica de su madera en construcciones en el pasado, abre la posibilidad de extender temporalmente los registros dendrocronológicos, en los Andes centrales de Perú.

Palabras claves: Dendrocronología, anillos de árboles, San Pedro Saño.

\begin{abstract}
The objective is to evaluate the Alnus acuminate potential for its later use in dendroclimatological studies. The research analyses the width tree-ring chronology for an A forest. "acuminate" in San Pedro de Saño and it was related the trees growth with the instrumental records of average, maximum and minimum temperature, precipitation and sunshine hours of the Huayao Weather Stations $\left(12^{\circ} 02^{\prime} 18.1^{\prime \prime}\right.$ S - $75^{\circ} 19^{\prime} 22.2^{\prime \prime}$ O) and Santa Ana (12 $12^{\circ} 00^{\prime} 15^{\prime \prime}$ $\left.\mathrm{S}-75^{\circ} 13^{\prime} 15^{\prime \prime} \mathrm{O}\right)$. The width ring chronology is composed of 33 sets of 22 trees and covered the 1968-2013 period. The trees growth was negatively related to the minimum monthly temperature of April this year. It was observed a positive and significant relationship between the growth timing and the Santa Ana station precipitation for March of the previous year and December of the current growth year. The April monthly sunshine hours of current year are significantly correlated with the radial growth. There is a precipitation influence of the previous summer and spring of the current year favoring the trees growth. The tree enters in dormancy, possibly due to the fall low temperatures. The wide range of distribution and the historic use of its wooden buildings in the past, opens the possibility to temporarily extend the dendrochronological records in the central Andes of Peru.
\end{abstract}

Keywords: Dendrochronology, tree-ring, San Pedro de Saño.

Historial del artículo:

Recibido: 1 de diciembre de 2014. Aprobado: 20 de noviembre de 2015. Disponible en línea: 30 de diciembre de 2015

1 Ingeniero Forestal y Ambiental, investigador de la Universidad Continental; profesor de Facultad de Ciencias Forestales y del Ambiente de la Universidad Nacional del Centro del Perú. 


\section{INTRODUCCIÓN}

La dendrocronología es la ciencia que estudia los anillos de crecimiento de los árboles para reconstruir, con resolución anual, eventos climáticos y procesos ecológicos pasados; esta técnica se aplica en especies que presentan anillos anuales bien diferenciados y un crecimiento radial influenciado por las fluctuaciones climáticas (1). La Dendrocronología es una valiosa herramienta para extender la serie de variables hidroclimáticas, ya que las variaciones interanuales en el espesor de crecimiento de los árboles están relacionadas con las fluctuaciones climáticas (1); por lo tanto, esta disciplina puede generar registros con una precisión anual, continuos en el tiempo, y que pueden llegar a extenderse eventualmente por miles de años. La dendrocronología es aplicada a diferentes disciplinas como, ecología, climatología, geología, hidrología y química, brindando información relevante en corto tiempo comparado con otros métodos tradicionales. La presencia de anillos de crecimiento anuales visibles es propia de la mayoría de especies arbóreas y arbustivas que se desarrollan en regiones templadas y frías donde existe una fuerte estacionalidad climática responsable de causar un período de dormancia o reposo en el crecimiento. Contrariamente, las regiones tropicales y subtropicales han sido tradicionalmente consideradas como regiones poco adecuadas para aplicar las técnicas dendrocronológicas tradicionales, debido a que en muchos casos los anillos de crecimiento no son claramente distinguibles en comparación con las especies de climas templados (2).

Las regiones subtropicales áridas y semiáridas de América del Sur, como el caso de los Andes centrales del Perú han sido escasamente investigadas desde este punto de vista. Sin embargo, en estos ambientes, las variaciones estacionales de la temperatura resultarían suficientes como para inducir en algunas especies la formación de anillos de crecimiento (3), aunado a ello la fuerte variación estacional en las precipitaciones (4). La cuenca del río Mantaro presenta una marcada estacionalidad con precipitaciones que se concentran entre octubre y febrero disminuyendo sus valores mínimos en junio y julio, coincidentemente en los meses que se registra los menores valores de temperaturas (4).

En regiones tropicales y subtropicales de los Andes de América se distribuye A. acuminata entre $12^{\circ} \mathrm{N}$ a $28^{\circ}$ $\mathrm{S}$ desde 600 - 3000 m s.n.m. (5). Tratándose de una de las principales especies arbóreas que crece en los Andes Centrales de Perú, es importante identificar la respuesta de $\mathrm{A}$. acuminata a los cambios climáticos a través del estudio de sus anillos de crecimiento, puesto que ya fue utilizado en otros estudios en otras latitudes (6). El objetivo de este trabajo fue evaluar el potencial de crecimiento $A$. acuminata para su uso posterior en estudios dendroclimatológicos; para lo cual se determina la correlación entre el crecimiento radial y las fluctuaciones climáticas de temperatura, precipitación y horas sol. La hipótesis involucrada es que $A$. acuminata presenta anillos distinguibles y una respuesta significativa a las variaciones climáticas.

\section{MATERIAL Y MÉTODOS}

Se utilizó un diseño descriptivo correlacional para conocer la asociación entre los índices de ancho de anillos con las variables climáticas de precipitación, temperatura media, temperatura máxima, temperatura

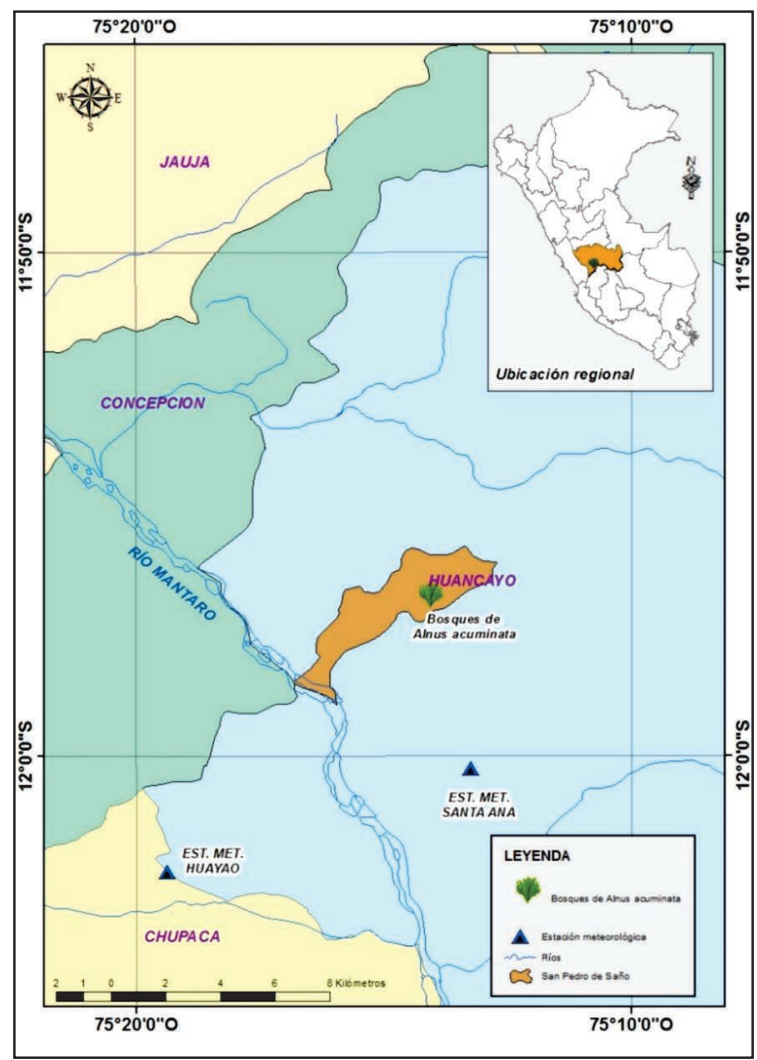

Figura $\mathrm{N}^{\circ} 1$ : Ubicación del bosque Alnus acuminata en Ilish Bichacoto y estaciones meteorológicas de donde se obtuvieron los datos climáticos.

mínima y horas sol.

Las muestras para este estudio fueron recolectados en el bosque reservado llish Bichacoto ubicada en el distrito de San Pedro de Saño (1 1 ${ }^{\circ} 56^{\prime} 50^{\prime \prime}$ S - $75^{\circ} 14^{\prime}$ 03" O) a 3636 m s.n.m. en una extensión de 120 ha; ecológicamente pertenece al bosque Seco Montano Bajo Subtropical (bs-MBS). El relieve es inclinado, típico de las laderas que encierran a valles interandinos. La biotemperatura media anual máxima es de $16,5^{\circ} \mathrm{C}$ y la media anual mínima de $10,9^{\circ} \mathrm{C}$. (7).

Toma de muestras: Los muestreos en la quebrada 
llish Bichacocto fueron realizados entre setiembre y noviembre de 2014. Los 45 árboles seleccionados fueron aquellos dominantes $y / o$ codominantes, de fuste cilíndrico y buen estado sanitario (8). Las muestras se tomaron con barrenos de incrementos, logrando secciones cilíndricas de $5 \mathrm{~mm}$ de diámetro y largo variable según el radio de cada muestra. De cada árbol se extrajeron y midieron dos radios.

Procesamiento de muestras: Se realizó el montado y preparación de las muestras para proceder a su fechado en el laboratorio de dendrocronología de la Facultad de Ciencias Forestales y del Ambiente de la Universidad Nacional del Centro del Perú. Las muestras extraídas con el barreno se fijaron con cola sobre regletas de madera acanaladas de aproximadamente un $\mathrm{cm}$ de espesor por dos de ancho, teniendo la precaución de que los elementos axiales del leño mantuvieran su posición vertical una vez montados. Esto facilitó la visibilidad de los anillos de crecimiento. Las muestras se dejaron secar a temperatura ambiente.
Determinación de la cronología de Alnus acuminata: Terminado el proceso de co-fechado, se midió el ancho de los anillos con un equipo velmex a una precisión de 0,001 mm, generándose series temporales para cada árbol. La calidad del fechado y de la medición fue controlada utilizando el programa Cofecha (12), que calcula índices de correlación entre las series individuales de anchos de anillos para identificar anillos ausentes y una evaluación de la fidelidad de los resultados, a partir de la utilización de ventanas de 35 años con una superposición de 17 años, con un coeficiente de correlación de 0,39 con $99 \%$ de confiabilidad. Las series de ancho de anillos resultantes de la medición de las bandas de crecimiento, fueron estandarizadas para eliminar las tendencias biológicas propias del crecimiento y minimizar las variaciones en el crecimiento que no son comunes a todos los árboles y que no están relacionadas con las variaciones climáticas (1). Las series fueron estandarizadas y promediadas para obtener una cronología final de sitio muestreado empleando el programa Arstan (13). Este

Tabla $N^{\circ}$ 1: Estaciones meteorológicas empleadas en este estudio.

\begin{tabular}{llcccc}
\hline \multicolumn{1}{c}{ Estación } & \multicolumn{1}{c}{ Variable } & $\begin{array}{c}\text { Periodo de } \\
\text { registro }\end{array}$ & $\begin{array}{c}\text { Latitud } \\
\left({ }^{\circ} \mathrm{S}\right)\end{array}$ & $\begin{array}{c}\text { Longitud } \\
\left({ }^{\circ} \mathrm{O}\right)\end{array}$ & Fuente \\
\hline Huayao & Precipitación & $1968-2009$ & $12^{\circ} 02^{\prime} 18.1^{\prime \prime}$ & $875^{\circ} 19^{\prime} 22.2^{\prime \prime}$ & IGP \\
Santa Ana & Precipitación & $1992-2013$ & $12^{\circ} 00^{\prime} 15^{\prime \prime}$ & $75^{\circ} 13^{\prime} 15^{\prime \prime} \mathrm{O}$ & SENAMHI \\
Huayao & Temperatura media & $1978-2008$ & $12^{\circ} 00^{\prime} 15^{\prime \prime}$ & $75^{\circ} 13^{\prime} 15^{\prime \prime} \mathrm{O}$ & SENAMHI \\
& Temperatura mínima & $1972-2008$ & $12^{\circ} 00^{\prime} 15^{\prime \prime}$ & $75^{\circ} 13^{\prime} 15^{\prime \prime} \mathrm{O}$ & SENAMHI \\
& Temperatura máxima & $1972-2008$ & $12^{\circ} 00^{\prime} 15^{\prime \prime}$ & $75^{\circ} 13^{\prime} 15^{\prime \prime} \mathrm{O}$ & SENAMHI \\
& Hora sol & $1972-2008$ & $12^{\circ} 00^{\prime} 15^{\prime \prime}$ & $75^{\circ} 13^{\prime} 15^{\prime \prime} \mathrm{O}$ & SENAMHI \\
\hline
\end{tabular}

SENAMHI: Servicio Nacional de Meteorología e Hidrología de Perú, IGP: Instituto Geofísico de Perú, Observatorio de Huancayo.

Posteriormente se procedió al pulido de las mismas utilizando lijas de diferente granulometría de 120 a 1000 progresivamente (9). Posteriormente los anillos de crecimiento fueron identificados y fechados bajo un estereoscopio marca Leica modelo S6E. Al anillo más joven recientemente formado se le asignó la fecha de acuerdo a Schulman (10) para el Hemisferio Sur, la que establece que la fecha del anillo más externo corresponde al año en que comenzó a formarse el anillo. Así, por ejemplo, la fecha del último anillo en las muestras tomadas en setiembre-noviembre de 2014, fue 2013, ya que se estima que ese anillo de crecimiento comenzó a formarse en octubre de 2013. Una vez asignada la fecha al anillo más externo, se continuó con el fechado hasta llegar al anillo más interno de la muestra. En cada muestra se marcó cada década con un punto, cada mitad de siglo con dos puntos. El co-fechado se realizó visualmente siguiendo las técnicas convencionales empleadas en dendrocronología (11), usando como referencia anillos más anchos y/o delgados. programa genera cronologías combinando las series estandarizadas de ancho de anillos con una media doblemente ponderada (13). Las series estandarizadas fueron promediadas obteniéndose una cronología media del sitio estudiado, constituyendo una serie temporal que representa las variaciones anuales en el crecimiento radial de los árboles de $\mathrm{A}$. acuminata.

Análisis entre el clima y el crecimiento radial: Con el objetivo de determinar las variables climáticas fuertemente relacionadas con el crecimiento de $\mathrm{A}$. acuminata en San Pedro de Saño, las variaciones interanuales en el ancho de anillos fueron comparadas con los registros instrumentales (horas sol, temperatura media, máxima, mínima y precipitación). Un método simple de comparación entre anillos de crecimiento y variaciones climáticas es el de la función de correlación (14). Este método consiste en correlacionar las variaciones interanuales en el ancho de los anillos de crecimiento de una cronología con las fluctuaciones climáticas interanuales tomadas 
mes por mes. La relación estadística entre el ancho de anillo y la variable climática es examinada sobre el período común entre la cronología y los datos instrumentales para cada variable; teniendo en cuenta que las condiciones climáticas durante la estación de crecimiento de un año dado influyen en el crecimiento cambial durante el siguiente ciclo vegetativo $(1,9)$, se muestreo y Huayao por poseer los registros climáticos más extensos. En la tabla 1 se describe las variables tomadas y los rangos de años utilizados.

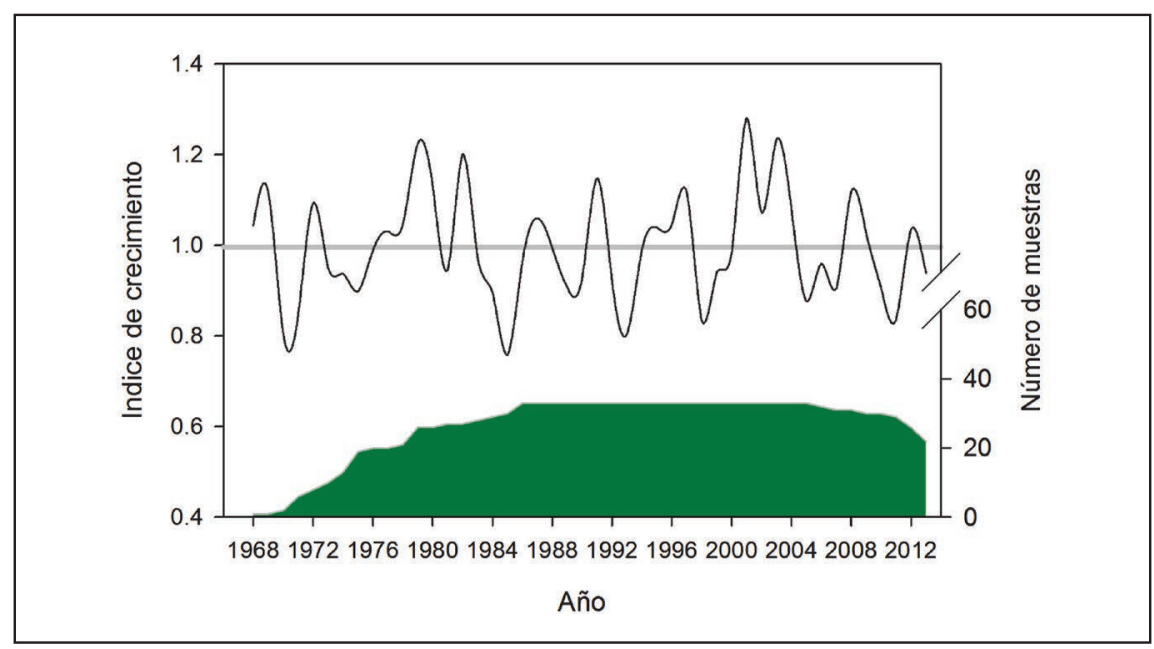

Figura $N^{\circ}$ 2: Cronología de ancho de anillos para Alnus acuminata para San Pedro de Saño. El área de color verde representa el número de muestras de la cronología.

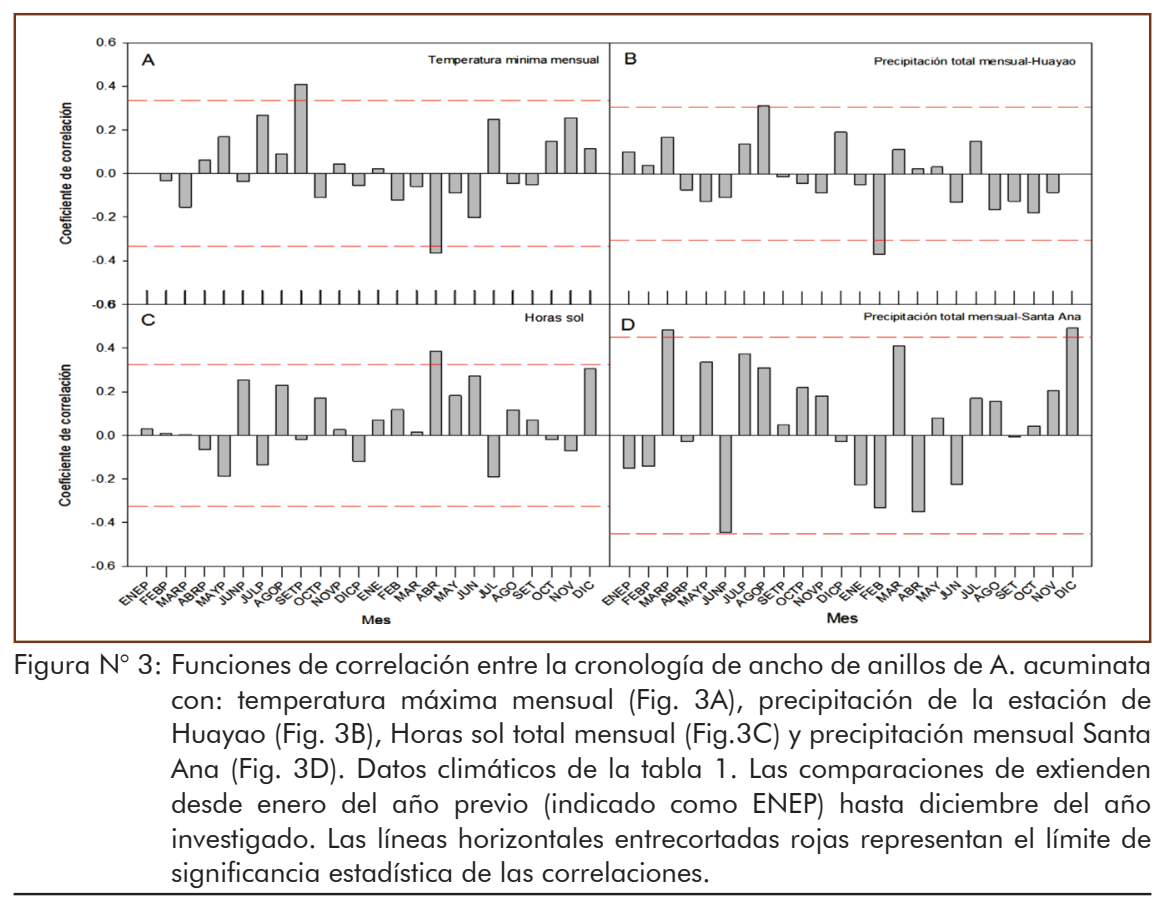

utilizó un periodo de 24 meses. De esta forma, se puede incluir en la comparación dos estaciones de crecimiento completas, la previa (crecimiento en el año " $x-1$ ") y la actual (crecimiento en el año " $x$ ").

Las estaciones meteorológicas utilizadas fueron la de Santa Ana por ser la más cercana al área de

\section{RESULTADOS}

Características de la cronología: A partir de las muestras obtenidas en el bosque Ilish Bichacoto, se logró fechar un $49 \%$ de los 45 árboles muestreados, obteniéndose una cronología de ancho de anillos de 
A. acuminata con una extensión de 46 años (19682013). Los estadísticos usados (15) para establecer la calidad de la cronología (desviación estándar $=0,12$, sensibilidad media $=0,12$ y la correlación media entre muestras $=0,26$ ), indican un alto porcentaje de señal común en las variaciones interanuales del crecimiento radial entre los individuos que conforman la cronología. La cronología está compuesta por 33 series de ancho de anillos correspondiente a 22 árboles. Con respecto al número de series por año, la cronología comienza en el año 1968 con una serie, aumenta a 8 en 1970 (figura 2, área color verde). Esto permite tener confiabilidad en los resultados a partir del año 1970, ya que existen 8 radios provenientes de 7 árboles diferentes. Las series de la cronología cuentan con un promedio de 38 años, con rangos que van entre un mínimo de 24 años y un máximo de 46 años. cronología de ancho de anillo y la precipitación del mes de diciembre del año investigado, se presenta la figura 4. Donde se observa que algunos años como por ejemplo en 1997 y 2001 existen altos valores de precipitación y los árboles responden con un mayor crecimiento radial. Por lo que se puede decir que las precipitaciones que caen al inicio del período de crecimiento favorecen el crecimiento radial.

\section{DISCUSIÓN}

Se presenta la primera aproximación a una cronología de ancho de anillos de 46 años de extensión, desarrollada a partir de Alnus acuminata para un bosque sobre los 3600 m s.n.m. en los andes centrales de Perú. Los resultados de este estudio indican que A. acuminata posee anillos de crecimiento anuales, pero

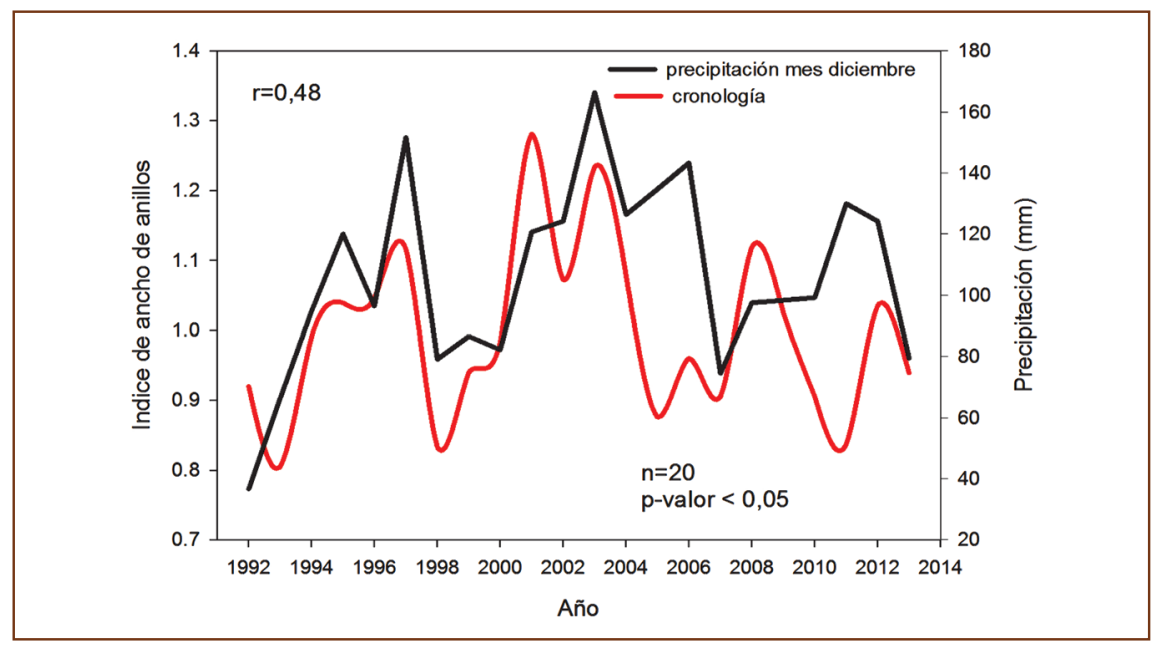

Figura $\mathrm{N}^{\circ}$ 4: Correlación entre cronología de ancho de anillos de A. acuminata y la precipitación del mes de diciembre de la estación meteorológica de Santa Ana.

Relación clima-crecimiento radial: La función de correlación muestra que el crecimiento radial de A. acuminata, se encuentra relacionado con las condiciones climáticas de temperatura mínima mensual de setiembre del año previo al $95 \%$ confianza $(r=$ 0,41 p-valor $<0,05$ ) y negativamente con las del mes de abril del año corriente $(r=-0,33$-valor $<0,05)$ figura 3A. En cuanto a la temperatura media y máxima mensual no se encontraron correlaciones significativas. Se observa una relación positiva y significativa entre la cronología de crecimiento y la precipitación de la estación de Santa Ana para los meses de marzo del año previo $(r=0,48 p$-valor $<0,05)$ y diciembre del año actual de crecimiento $(r=0,48 p<0,05)$ figura 3D. Las horas sol mensuales de abril del año corriente tienen una correlación significativa con el crecimiento radial $(r=0,39 p$-valor $<0,05)$ figura 3C.

Sobre la base de la correlación significativa entre la las muestras extraídas con barreno no ayudan a definir si están claramente demarcados.

Debido al poco campo de amplitud visual $(5 \mathrm{~mm}$ de diámetro) de las muestras extraídas con el barreno de incremento, no se pudieron delimitar bien los anillos de crecimiento. Por lo cual se sugiere para futuros estudios, utilizar secciones transversales de árboles $(16,17)$, porque brinda mayor campo de observación de la muestra y facilita la identificación de los anillos de crecimiento anuales.

Para desarrollar la cronología de ancho de anillos se utilizó el $49 \%$ de los árboles muestreados (22 de un total de 45 árboles). El resto de las series medidas que no integraron la cronología, no pudieron ser datados correctamente por el poco campo visual y debido a que podrían presentar anomalías en el crecimiento, tales como: presencia de lentes, anillos indiferenciados 
y anillos falsos que no permitieron lograr la datación correcta de las muestras. Estos resultados del porcentaje de árboles empleados están por debajo de otros estudios previos de A. acuminata en Argentina (18) y otras especies desarrolladas en los Andes de Sudamérica $(9,19,20)$.

La escala temporal de la cronología es corta en comparación con las cronologías mayores de 100 años obtenidos en la provincia biogeográfica de las corriente favorecen al crecimiento del árbol, indicando que posiblemente la precipitación es uno de los factores que controla el crecimiento (figura 3D). Las correlaciones negativas con la temperatura mínima en otoño, estarían reflejando que en esta época el árbol entra en dormancia, formando madera tardía. No queda claro el mecanismo que explique, las relaciones positivas entre las horas sol de otoño del año corriente con el ancho de anillos. Una posible respuesta podría ser que, el otro factor que controla el crecimiento de

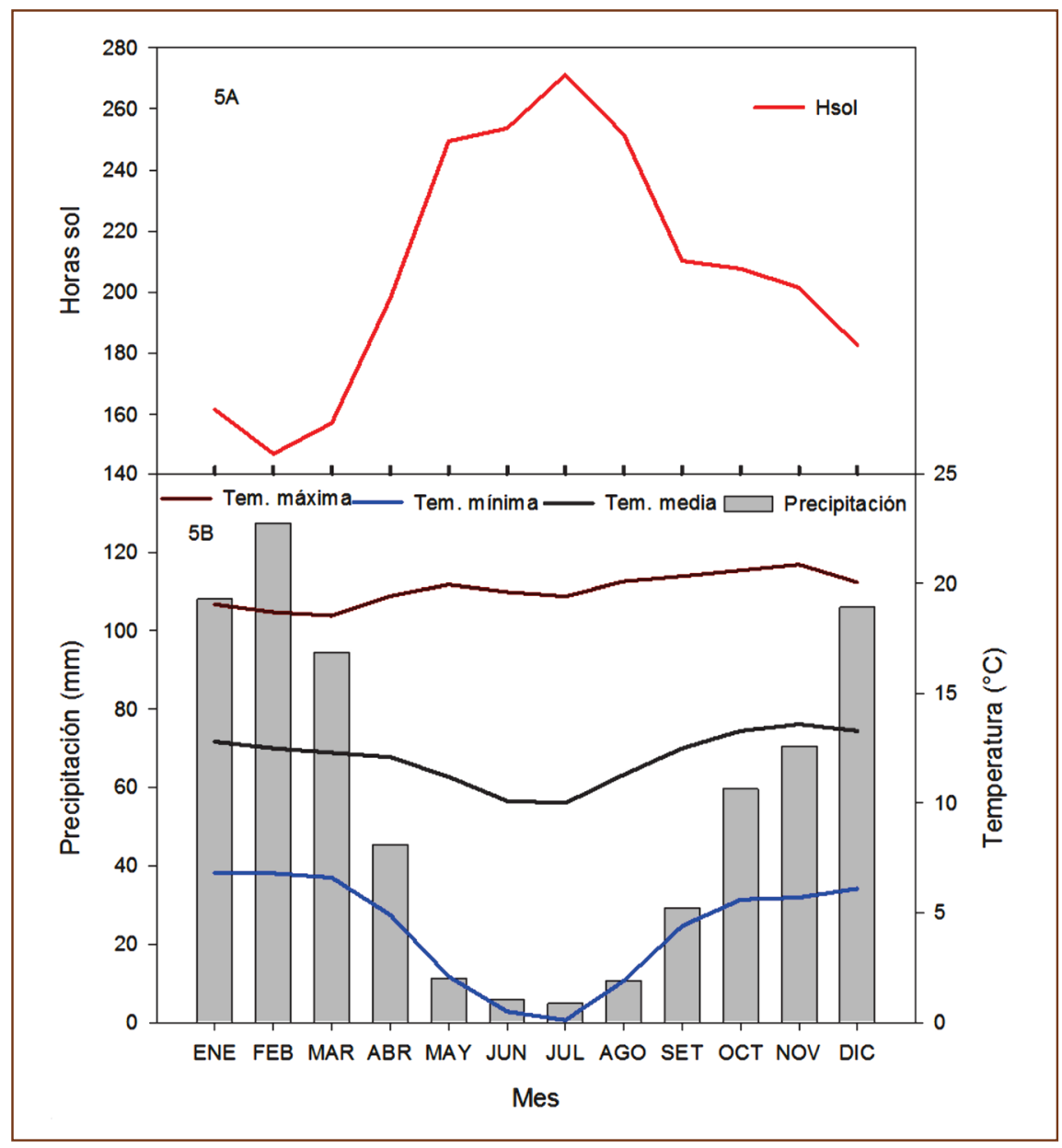

Figura $\mathrm{N}^{\circ}$ 5: Horas sol mensual de la estación de Huayao (5A). Climatología de precipitación correspondiente a la estación meteorológica de Santa Ana y de temperatura a estación de Huayao (5B).

Yungas en el nor oeste de Argentina (18). A pesar de la corta escala temporal de la cronología de ancho de anillos para San Pedro de Saño, se puede aplicar la dendroecología para estudios de dinámica de bosques de A. acuminata.

Las funciones de correlación indican que el crecimiento de A. acuminata está regulado por las variaciones climáticas del valle del Mantaro. En general, las precipitaciones del verano previo y primavera del año
A. acuminata en estos lugares es la escorrentía, puesto que esta especie crece muy cercano a los cauces de riachuelos, donde la disponibilidad de agua está presente casi todo el año (1).

El amplio rango de distribución de la especie, la marcada estacionalidad de la precipitación, con más de tres meses seguidos de precipitación menor a $20 \mathrm{~mm}$ lo que coincide con los registros de las temperaturas bajas con alta radiación solar contribuyen a la 
formación de leño tardío, formándose el límite de los anillos de crecimiento (figura 5) y la utilización histórica de su madera en construcciones en el pasado. Es así, que se abre la posibilidad de extender temporal y espacialmente los registros dendrocronológicos, en los andes centrales de Perú, cubriendo un amplio espacio, donde hasta la última década no existe registros de cronologías desarrolladas (figura 6).

La cronología desarrollada demuestra el potencial de esta especie para ser utilizada con fines dendroclimáticos porque presentan anillos diferenciados y están influenciados por variables climáticas de temperatura mínima y precipitación. Esta situación

\section{REFERENCIAS BIBLIOGRÁFICAS}

1. Fritts HC. Tree-ring and climate. London: Academic Press; 1976.

2. Worbes $M$. One hundred years of tree ring research in the tropics. - A brief history and an outlook to future challenges. Dendrochronologia. 2002; 20(1-2): 217-231.

3. Villalba R, Grau HR, Bonisegna J, et al. Climatic variations in subtropical South America inferred from upper-elevation tree-ring records. International Journal of Climatology. 1998;

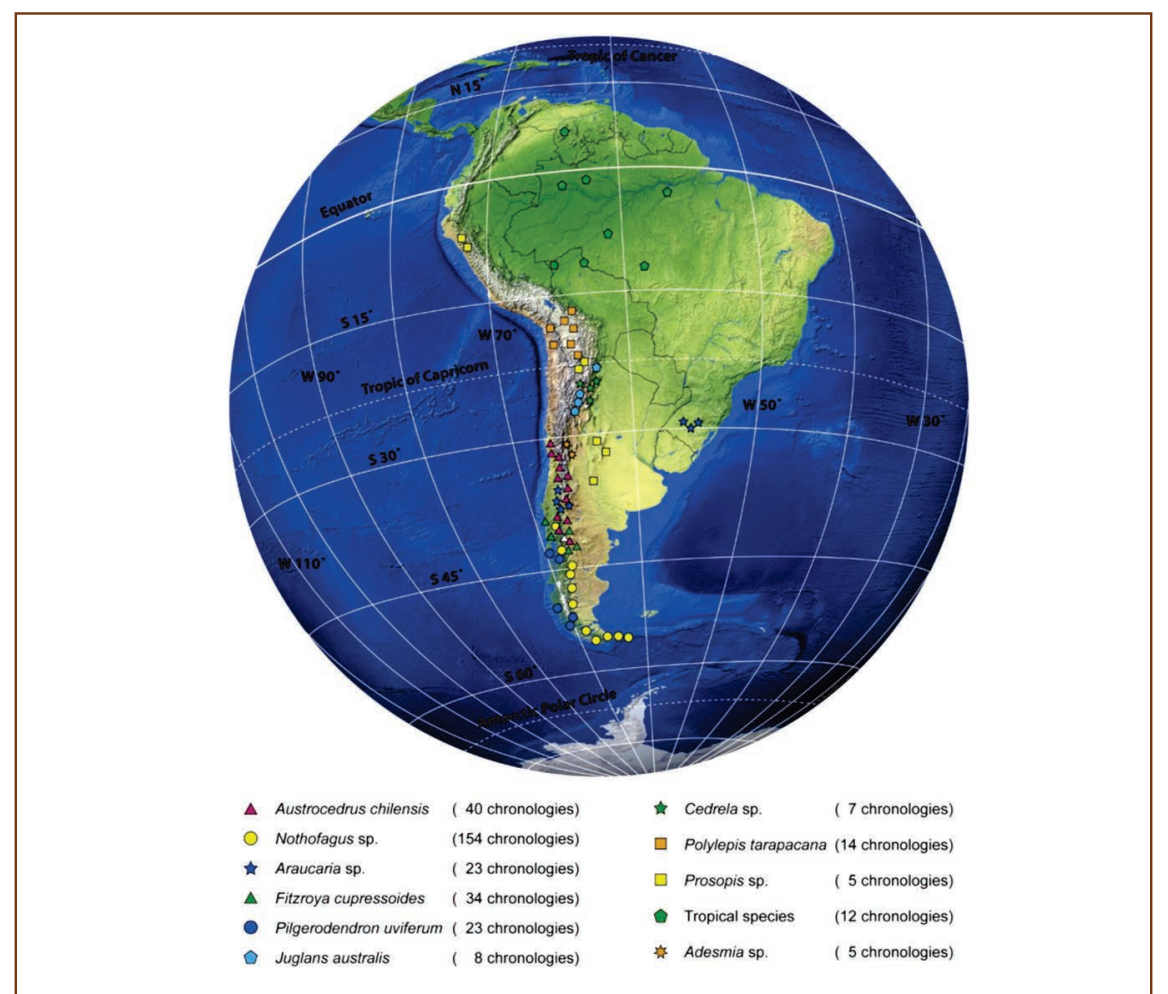

Figura $\mathrm{N}^{\circ}$ 6: Cronologías de anillos de árboles en Sudamérica. Cada símbolo representa la ubicación aproximada de las cronologías. El número de cronologías compiladas para diferentes especies es indicado entre paréntesis. Fuente: (21).

más la posibilidad de extraer material del antiguas construcciones como iglesias, casas; abre la posibilidad para el desarrollo de futuras cronologías y posteriores reconstrucciones climáticas.

\section{Agradecimientos}

Este trabajo se realizó gracias al apoyo económico de fomento de investigación de la Universidad Continental. A los estudiantes del curso de Ecología, por el apoyo en la colecta de muestras. A Rosana Ordaya por las inagotables horas de muestreo.

\section{8(13): 1463-1478.}

4. Instituto Geofísico del Perú. Atlas Climático de precipitación y temperatura del aire de la Cuenca del Río Mantaro. Lima: CONAM / IGP; 2005.

5. Killen TJ, Garcia E, Beck S. Guía de Arboles de Bolivia. Bolivia: Herbario de Bolivia and Missouri Botanical Garden; 1993.

6. Morales M, Villalba R, Grau R, et al. Rainfallcontrolled tree growth in high elevation subtropical treelines. Ecology. 2004; 85(1 1): 3080-3089.

7. Administración Local de Agua - Mantaro. Evaluación de los recursos hídricos superficiales en la cuenca río Mantaro. Lima; 2010. Disponible en: http://www.ana.gob.pe/sites/default/files/ 
publication/files/evaluacion_rh_superficiales_rio_ mantaro_0.pdf

8. Villanueva J, Cerano J, Constante V, et al. Muestreo Dendrocronológico: colecta, preparación y procesamiento de núcleos de crecimiento y secciones transversales. Durango: Grupo Colorama; 2009.

9. Argollo M, Solíz C, Villalba R. Potencialidad dendrocronológica de Polylepis tarapacana en los Andes centrales de Bolivia. Ecologia en Boliviana. 2004; 39(1): 5-24.

10. Schulman E. Dendroclimatic changes in semiarid America. Tucson: University of Arizona Press; 1956.

11. Stokes MA, Smiley TL. An introduction to tree-ring dating. Chicago: University of Chicago Press; 1968.

12. Holmes RL. Computer-assisted quality control in tree-ring dating and measurement. Tree-Ring Bulletin. 1983; 43: 69-75.

13. Cook ER. A time series analysis approach to treering standardization. [Tesis doctoral]. Tucson: The University of Arizona; 1985.

14. Blasing TJ, Solomón AM, Duvick DN. Response functions revisited. Tree-Ring Bulletin. 1984; 44: $1-15$.

15. Briffa KR. Interpreting High-Resolution Proxy Climate Data - The Example of Dendroclimatology. In: Storch V, Navarra HA, editors. Analysis of
Climate Variability, Applications of Statistical Techniques. Berlin: Springer; 1995.

16. López L. Villalba R. Climate Influences on the Radial Growth of Centrolobium microchaete, a valuable Timber Species from the Tropical Dry Forests in Bolivia. Biotropica. 2011; 43(1): 41-49.

17. Pereyra $M$, Inga G, Morales $M$, et al. Potencialidad de Cedrela odorata (Meliaceae) para estudios dendrocronológicos en la selva central del Perú. Biotropica. 2014; 62(2): 783-793.

18. Grau HR, Easlade TA, Paolini L. Subtropical dendroecology - dating disturbances and forest dinamics in northwestern Argentine montane ecosystems. Forest Ecology Management. 2003; 177: 131-143.

19. Moya J. \& Lara A. Cronologías de ancho de anillos de queñoa (Polylepis tarapacana) para los últimos 500 años en el Altiplano de la región de Arica y Parinacota, Chile. Bosque. 2011 ; 32(2): 165-173.

20. Morales M, Christie DA, Villalba $R$, et al. Precipitation changes in the South American Altiplano since 1300AD reconstructed by treerings. Climate of the Past. 2012; 8: 653-666.

21. Boninsegma JA, Argollo J, Aravena JC, et al. Dendroclimatological reconstructions in South America: A review. Palaegeography, Paleoclimatology, Palaeoecology. 2009; 281: 210-228. 